\title{
Approche de la diversité des systèmes d'élevage laitiers à la Réunion
}

\author{
V. Alary ${ }^{1,2}$ S. Messad ${ }^{1}$ C. Taché ${ }^{3}$ E. Tillard ${ }^{4}$
}

\begin{abstract}
Mots-clés
Classification - Système d'exploitation agricole - Analyse factorielle Elevage - Lait - Réunion.
\end{abstract}

\begin{abstract}
Résumé
Les effets de changements de l'environnement économique, réglementaire et technique des exploitations sur leur fonctionnement et leurs performances diffèrent en fonction des dotations initiales des exploitants, de leur projet, des modes d'organisation et de conduite des systèmes productifs, des possibilités d'investissement, etc. Cependant, il est difficile de saisir l'exhaustivité des cas et seule la construction d'une typologie peut permettre de saisir la diversité des exploitations pour approcher les effets différenciés de ces changements, comme pour ajuster la recherche et le développement aux besoins et aux structures variées dans un territoire donné. La typologie réalisée pour caractériser la diversité des exploitations laitières à la Réunion résulte d'un processus de construction itératif qui s'est appuyé sur certains principes de la typologie à dire d'expert en recourant aux méthodes mutivariées. Cette typologie est construite sur la base d'enquêtes et d'entretiens réalisés auprès des éleveurs et des différents partenaires de la filière : coopératives, agences de développement, autorités locales, centre de gestion.
\end{abstract}

\section{- INTRODUCTION}

Les effets de changements de l'environnement économique, réglementaire et technique sur le fonctionnement et les performances technico-économiques des exploitations diffèrent généralement en fonction des dotations initiales des exploitants (cheptel vivant, foncier, travailleurs familiaux ou salariés), de leur projet, des modes d'organisation et de conduite des systèmes productifs, des possibilités d'investissement, etc. Les activités non agricoles par la mobilisation de main d'œuvre comme la génération de revenu au sein de l'exploitation peuvent freiner le processus d'adaptation comme

\footnotetext{
1. Cirad-emvt, TA 30/A, Campus international de Baillarguet, 34398 Montpellier Cedex 5, France

2. Icarda, 6 rue Ibn Rach, 2080 Nouvelle Ariana, Tunisie E-mail : veronique.alary@ cirad.fr

3. Ensar, 65 rue de St Brieuc, 35042 Rennes Cedex, France

4. Cirad-emvt, station de Ligne-Paradis, 7 chemin de l'Irat, Ligne Paradis, 97410 Saint-Pierre, Réunion
}

faciliter les changements, fournissant à la fois sécurité et moyens monétaires. Dès lors, l'exploitation est un système complexe dont la gestion familiale comme l'histoire des hommes créent souvent un certain particularisme dans le fonctionnement global. Cependant, il est difficile d'étudier l'exhaustivité des cas et seule la construction d'une typologie peut permettre de saisir la diversité des exploitations, pour approcher les effets différenciés de changements extérieurs, comme ajuster la recherche et le développement aux besoins et aux structures variées dans un territoire donné. En effet, ces approches typologiques ont l'insigne avantage de fournir un ensemble de données de référence aux agents du développement pour apprécier la teneur des changements actuels et d'ajuster la formulation du conseil technico-économique aux exploitations.

Ainsi, à la Réunion, à la demande de la coopérative laitière (Sica Lait) et avec le concours de différents agents du développement (EDE ${ }^{1}, \mathrm{U}-\mathrm{APF}{ }^{2}$, Centre de gestion), un projet de recherche et

\footnotetext{
${ }^{1}$ Etablissement département élevage

${ }^{2}$ Union des Associations foncières pastorales
} 
développement Cirad-pôle élevage a été initié en 2000 pour approcher et évaluer les effets à court et moyen termes d'événements divers (modification des prix, des aides, des règlements, innovation technique) sur les systèmes d'élevage laitier. Dans un contexte de saturation foncière et de remise en cause du modèle productiviste dans la société, la demande d'installation d'agriculteurs sur un espace restreint pose aussi des problèmes de viabilité économique et d'acceptabilité sociale. Face à ces enjeux, l'analyse typologique doit permettre d'identifier des exploitations type, caractérisant la diversité des modes de fonctionnement des exploitations, qui serviront de référence pour approcher les effets de changements technologiques ou institutionnels, et ce, à l'aide d'un modèle de programmation mathématique à l'échelle de ces exploitations types. Aussi, la typologie est une étape dans le processus d'identification et de caractérisation des pôles de développement de l'élevage laitier (ou encore des exploitations de référence), pour alimenter des modèles d'exploitation nécessaires aux simulations dynamiques. Dans un objectif de représentativité de la totalité des exploitations réunionnaise, cette étape permet aussi de poser les bases d'une typologie «à dire d'expert ».

L'approche typologique, bien ancrée dans l'analyse du fonctionnement des exploitations en France, a conduit à de nombreux développements méthodologiques (25). On peut citer deux grandes familles : les typologies à dire d'expert et les typologies élaborées à partir d'enquêtes réalisées en exploitation dont le traitement s'appuie sur des analyses multivariées. La typologie réalisée à la Réunion résulte d'un processus de construction itératif qui s'est appuyé sur certains principes de la typologie à dire d'expert en recourant aux méthodes mutivariées. Cette typologie est construite sur la base d'enquêtes et d'entretiens réalisés auprès des éleveurs et des différents partenaires de la filière. Il s'agira donc de présenter, à ce stade du processus de caractérisation des exploitations, la méthode utilisée pour approcher et caractériser la diversité des exploitations laitières à la Réunion et de voir comment la typologie permet de répondre à des questions de développement comme la viabilité différenciée des exploitations selon le degré d'intensification.

\section{MATERIEL ET METHODES}

\section{Evolution des approches typologiques des exploitations}

Les typologies d'exploitations agricoles se sont progressivement construites et inscrites dans une démarche de recherche et de diagnostic du fonctionnement des exploitations, qui s'appuie sur un corpus conceptuel relatif à la modélisation systémique du fonctionnement global de l'exploitation, de sa trajectoire d'évolution, du système famille-exploitation $(3,16,23)$. Les typologies répondent à différentes questions : cadre d'analyse pour l'étude de problèmes techniques, formulation de conseil technico-économique aux exploitants, étude prospective d'impacts de changements de politiques économiques ou de changements techniques. L'avantage de l'approche fonctionnelle ou globale des exploitations est de pouvoir resituer une problématique de développement ou de recherche dans un cadre commun pluridisciplinaire pour les chercheurs ou pour les agents du développement $(8,17)$.

Les méthodes typologiques ont connu des évolutions remettant peu en cause les concepts de trajectoire d'évolution ou de fonctionnement global des exploitations, mais davantage l'approche méthodologique. Une des premières méthodes a été développée à la fin des années 1970 et au début des années 1980 pour accompagner la démarche de l'approche globale du fonctionnement des exploitations (4). Elle s'appuie sur un système d'enquêtes approfondies sur un échantillon raisonné d'exploitations et résulte de comparaisons
« de proche en proche » sur les classes voisines de fonctionnement permettant le regroupement en types. Ces types, définis par un ensemble d'éléments discriminants, permettent d'organiser la production de références en fonction de la diversité des situations, mais aussi d'identifier des trajectoires d'évolution (5).

Face à une demande sociale évolutive en termes d'opérationnalité des typologies (mise au point de procédure d'actualisation, typologie à des fins prospectives, prise en compte des nouvelles fonctions de l'agriculture, notamment environnementales), de nouvelles méthodes ont été développées (17). Une première méthode issue de l'approche classique est basée sur une analyse comparative conduite par itération qui permet de définir un ensemble de fonctions qui discriminent le mieux les systèmes d'exploitations ; ces fonctions combinées déterminent des axes multivariés qui constituent alors une grille d'analyse où se positionnent les types d'exploitations, définis par un ensemble organisé de critères discriminants. La représentation graphique de la grille d'analyse permet d'identifier des groupes qui structurent la population étudiée et des filiations entre groupes voisins. Une deuxième méthode (à dire d'expert) s'est développée dans un objectif de fournir une méthodologie utilisable par les différents intervenants du milieu agricole, sans recourir à des enquêtes approfondies et en s'appuyant sur des connaissances préexistantes. Le principe fondamental de cette méthode est la construction de types «par agrégation autour de pôles virtuels définis à dire d'experts » $(25,26,27)$. Chaque type est ensuite décrit à partir de l'analyse statistique des données disponibles. Autrement dit, il ne s'agit plus de rechercher les facteurs discriminants de la population, mais davantage de mesurer le degré de ressemblance d'une exploitation à un type construit à partir de la connaissance des experts du terrain. En conséquence, les types construits ne changent pas au cours du temps, même si de nouveaux types peuvent être élaborés ou certains supprimés. En revanche, les individus peuvent évoluer d'un type à l'autre.

Ces deux approches typologiques s'opposeraient en apparence aux méthodes de classification issues des analyses statistiques multivariées dont le principe est de raisonner sur un nombre quelconque d'objets à classer, sans a priori ou hiérarchie sur les objets. Les principaux inconvénients des méthodes statistiques seraient de deux ordres : (i) le choix arbitraire des modalités ou variables pour caractériser les types ; (ii) la non-prise en compte de la dimension historique. S'il est certes difficile de dégager des trajectoires d'évolution à partir d'une typologie instantanée, on peut toutefois émettre des hypothèses sur des logiques d'évolution ou du moins dégager des filiations entre types à partir de l'analyse des proximités des types sur le plan factoriel. En outre, il est difficile pour l'individu de prendre en considération l'ensemble des facteurs pour expliquer un fonctionnement global et l'analyse statistique multivariée présente l'avantage de travailler sur un nombre quelconque de données et ainsi de saisir la complexité des systèmes d'exploitations. Cette méthode permet, dès lors, de mettre en évidence des liens entre fonctions ou critères peu apparents a priori. Ou a contrario de reléguer au second plan un facteur qui semblait a priori déterminant des modes de fonctionnement. Dans les pays du Sud, ces approches constituent un moyen d'aborder la diversité et la complexité des systèmes pour analyser la place de l'élevage à l'intérieur des systèmes d'exploitation et comprendre la cohérence des choix et des actions dans leur globalité $(20,22)$. Par ailleurs, ces approches sont aussi souvent la base pour la construction d'un réseau de fermes de référence en vue de la mise en place de suivi de performances zootechniques ou sanitaires ou, plus généralement, le développement de projets de recherche et développement englobant la diversité des cas $(14,21)$.

Cependant, ces avantages ne doivent pas faire oublier le caractère parfois arbitraire du choix des variables qui conditionne les types 
obtenus. A ce titre, l'analyse factorielle multiple (Afm) présente l'avantage de raisonner sur un ensemble de variables reflétant une fonction ou des attributs discriminants, définis à partir de la connaissance du terrain. En outre, l'étude comparée des fonctions ou attributs autorise des analyses en termes de corrélation ou encore de cause à effet (13) et la position des individus pour les fonctions ou thèmes donnés permet d'approcher plus facilement la diversité intra-groupe et d'émettre des hypothèses d'évolution. Les auteurs ont ainsi étudié la diversité des exploitations laitières de la Réunion à l'aide de l'Afm.

\section{Contexte de l'approche typologique raisonnée des élevages laitiers à la Réunion}

L'élevage laitier à la Réunion s'est développé dans les années 1960-70 grâce aux politiques de développement économique et social des Hauts de l'île et notamment à la mise en place du Plan d'aménagement des Hauts (réforme foncière, développement d'infrastructure, aide à la création de prairies, etc.). Les années 1980 ont été marquées par le développement d'un ensemble de soutien spécifique à l'activité laitière, notamment grâce à la création du Fonds de développement laitier qui a servi au soutien du prix du lait, au financement des opérations de collecte, de qualité du lait, etc., mais aussi à la mise en place des Plans de développement laitier ( $\mathrm{Pdl})$ pour favoriser les investissements agricoles. Les événements conjugués du début des années 1990 (changement du mode de collecte, arrivée d'un nouveau transformateur, crise de la dermatose, mise en place du revenu minimum pour les sansemploi) ont été les stimulants d'un nouveau développement de l'activité laitière, basé selon un modèle qui se veut à la fois viable, reproductible et orienté vers la production. Ainsi, les nouveaux plans de développement laitier visent aujourd'hui la mise en place d'exploitations comptant un minimum de 30 vaches laitières.

La Sica lait compte aujourd'hui près de 150 adhérents. Ce nombre limité explique qu'un suivi rapproché des éleveurs est possible, à la fois par les techniciens de la Sica Lait, mais aussi par les différents partenaires de la filière. En outre, depuis une quinzaine d'années, le Cirad a mis en place des suivis auprès des éleveurs qui permettent aujourd'hui d'avoir une bonne connaissance de l'évolution des différents types d'élevages laitiers en fonction de leur localisation géographique qui conditionne les modes d'utilisation de l'espace (2), de leurs performances (30) et des changements techniques, notamment en matière d'affouragement (24). C'est donc sur la base de ce corpus de connaissances que s'est mise en place l'analyse du fonctionnement des exploitations. Compte tenu des finalités multiples de cette typologie (approche de la viabilité économique des exploitations, constitution d'une base de références technico-économiques, construction de modèles d'exploitation en vue de leur formalisation dans un outil d'aide à la décision), la démarche a visé l'élaboration d'une typologie de fonctionnement global des exploitations pour avoir une situation des exploitations au temps $t$ et comprendre les principaux choix d'orientation des exploitants, selon les contraintes environnementales, techniques et économiques.

Le fonctionnement des exploitations a été appréhendé par un système d'enquêtes individuelles auprès des exploitants, d'entretiens auprès des différents partenaires de la filière lait (coopérative, administration, Centre de gestion, etc.) et de restitution des résultats en groupe afin de les valider ou de les confronter à nouveau à la réalité du terrain. Une première enquête a été conduite auprès des 20 exploitations en suivi de performance au Cirad, choisies selon des critères géographiques et de performances zootechniques. Il s'agissait de confirmer, de rectifier ou d'approfondir les principaux critères de différenciation des élevages définis par les agents ou techniciens du développement et discutés lors d'une réunion de groupe, c'est-à-dire : la vitesse de croissance du cheptel depuis 10 ans, la stratégie d'affouragement, la préparation de la succession ou non. Cette enquête auprès des chefs d'exploitation a été axée sur l'histoire de la famille et de l'exploitation (constitution du cheptel, du foncier, etc.) depuis son installation, les principaux systèmes d'affouragement et les projets des éleveurs en matière d'élevage, de succession et de diversification. Parallèlement, ont été collectées les données de suivi technico-économique et comptable auprès de l'EDE et du Centre de gestion. A partir de cet ensemble de données, une première typologie a été réalisée à l'aide de méthodes multivariées. Le choix des variables a résulté essentiellement des hypothèses de regroupement émises par les agents de développement. Cette première typologie a permis à la fois de détecter l'absence de certaines catégories d'exploitation - pourtant identifiées par les agents de la filière - mais aussi d'affiner, voire de reconsidérer, les types définis à dire d'expert, lors des entretiens de groupe avec les agents du secteur laitier. Ainsi, ont été identifiées de nouvelles exploitations à enquêter, conduisant à un échantillon final de 32 exploitations, soit 20 p. 100 de la population des éleveurs laitiers de l'île. A l'enquête préliminaire s'est ajoutée une enquête approfondie permettant de comprendre les liens entre les résultats technico-économiques ou comptables et les stratégies des éleveurs identifiées dans le premier questionnaire. Cette dernière enquête a été axée sur les pratiques de gestion du troupeau et de l'alimentation.

L'analyse typologique séparée des données socio-structurelles et économiques collectées en 2000 sur 32 exploitations laitières a permis d'identifier des groupes d'exploitations laitières par un ensemble de variables très disparates (28). Les principaux facteurs de différenciation socio-structurelle ont été le degré de capitalisation (taille du cheptel, état du foncier) et la vitesse de constitution de ce capital (aspects historiques) ; la typologie économique a distingué les exploitations en fonction des résultats d'exploitation (proportionnel au degré de capitalisation) et du niveau d'endettement (relié à l'histoire de l'exploitation). Cependant, ce travail a abouti à deux typologies qui ne se juxtaposaient pas. Des petites exploitations se trouvaient appartenir à deux groupes économiques différents en fonction du mode de gestion du troupeau, de la maîtrise du processus de production ou encore du stade de développement. Des exploitations pouvaient avoir des résultats d'exploitation relativement proches alors qu'elles divergeaient dans les modes de fonctionnement. Il devenait alors difficile de saisir à la fois la complexité et la cohérence entre les groupes.

Les difficultés d'interprétation des résultats ont conduit à travailler sur des groupes de variables ou de modalités reflétant une certaine fonction, situation ou évolution d'une partie ou de toute l'exploitation. Ces ensembles de variables ou modalités sont appelés des thèmes. Le choix des thèmes suppose la définition de souscomposants d'un système global. Partant de l'approche systémique des exploitations, qui offre une représentation à la fois synthétique et cohérente du mode de fonctionnement de l'exploitation, ont été définis les thèmes de "système fourrager », " conduite du cheptel» et le système de pilotage qui comprend « historique de l'exploitation » et «projets et autres activités ». Cependant, ont été distingués les thèmes tels que " charges d'alimentation ", « contraintes » (relatives à la zone) et « transactions » (achat et vente de fourrages) pour appréhender le degré d'autonomie fourragère ou alimentaire, défini comme un élément clé de différenciation par la coopérative (tableau I).

Dès lors, les thèmes utilisés dans l'analyse multivariée ont été construits à partir : (i) de l'analyse système du fonctionnement des exploitations visant à comprendre le fonctionnement de l'exploitation selon les dotations initiales, l'histoire de l'exploitant, les choix et les itinéraires de production, les projets dans et hors exploitation 


\section{Tableau I}

Structuration en thèmes des informations collectées sur les exploitations

\begin{tabular}{|c|c|}
\hline Thèmes & Composantes \\
\hline \multicolumn{2}{|c|}{ Structure et fonctionnement des exploitations } \\
\hline Capital de l'exploitation & $\begin{array}{l}\text { Social, foncier, cheptel, aides } \\
\text { à l'investissement }\end{array}$ \\
\hline Système fourrager & $\begin{array}{l}\text { Allocation de la terre, } \\
\text { mode de récolte }\end{array}$ \\
\hline Conduite du cheptel & $\begin{array}{l}\text { Structure, chargement, } \\
\text { production, alimentation }\end{array}$ \\
\hline Contraintes & $\begin{array}{l}\text { Zones, ressources fourragères, } \\
\text { main d'œuvre }\end{array}$ \\
\hline Transactions & Fourrages, lait, animaux \\
\hline Historique de l'exploitation & $\begin{array}{l}\text { Caractéristiques du chef } \\
\text { d'exploitation, histoire de } \\
\text { l'exploitation }\end{array}$ \\
\hline Projets et autres activités & $\begin{array}{l}\text { Souhait familial } \\
\text { et professionnel }\end{array}$ \\
\hline \multicolumn{2}{|l|}{ Résultats économiques } \\
\hline Budget & $\begin{array}{l}\text { Charges, subventions, } \\
\text { trésorerie }\end{array}$ \\
\hline Charges d'alimentation & $\begin{array}{l}\text { Structure des charges } \\
\text { d'alimentation }\end{array}$ \\
\hline Résultats & $\begin{array}{l}\text { Marge brute, excédent brut } \\
\text { d'exploitation, valeur ajoutée, } \\
\text { productivité }\end{array}$ \\
\hline Bilan & Structure actif/passif \\
\hline Endettement & $\begin{array}{l}\text { Structure des emprunts, } \\
\text { dépendance financière }\end{array}$ \\
\hline
\end{tabular}

(reflétant les objectifs des éleveurs à moyen et long termes) ; (ii) d'une analyse des comptes d'exploitation reflétant les modes de gestion de trésorerie à court terme et la solidité de l'exploitation à plus ou moins long terme ; (iii) des critères de différentiation à dire d'expert. L'analyse comparée des thèmes doit permettre de comprendre la cohérence entre les choix de production, les décisions d'investissement et les projets à moyen-long terme, par rapport aux résultats technico-économiques. En effet, le fonctionnement de l'exploitation résulte justement de l'articulation d'un ensemble de données (données de structure comme la taille de l'exploitation, données contraignantes comme le climat et les caractéristiques physiques du sol, données sociales comme le retour d'un fils et l'origine du chef d'exploitation, etc.) qui, à un moment donné de la vie de l'exploitation, donne une image cohérente de la situation, des choix et des actions de ce système. Isoler ou identifier des sous-composantes revient donc à effectuer un découpage fictif au temps t.

\section{L'analyse factorielle multiple comme outil d'analyse de la diversité}

L'Afm fait partie des méthodes factorielles multitableaux. Elle permet de mettre en évidence les relations existant entre des variables regroupées en blocs, en recherchant les facteurs communs de différenciation des individus statistiques. Cette recherche peut se subdiviser en deux sous-analyses : (i) une analyse globale (interstructure) qui aide à évaluer les corrélations entre les différents groupes de variables sans aller dans le détail des éléments qui les différencient ; (ii) une analyse fine (intra-structure) qui fournit des éléments d'explication des différences et des ressemblances entre ces groupes.

Les aspects théoriques de la méthode ont fait l'objet de nombreux travaux de recherche et n'ont donc pas été détaillés $(9,10,12,15$, 18). Dans cette étude, les auteurs se sont limités à une présentation simplifiée du principe et de la démarche analytique de l'Afm.

La structure des tableaux multiples est celle que l'on rencontre en pratique dans les enquêtes. Il s'agit d'un tableau $X$ à $n$ individus (les exploitations) et $p$ variables (les questions des enquêtes, les éléments des comptes et des bilans d'exploitation) résultant de la juxtaposition de $K$ tableaux à $m_{k}$ variables tels que :

$X=\left(X_{1}, \ldots X_{i}, \ldots X_{k}\right)$ et $p=m_{1}+\ldots+m_{i}+\ldots+m_{k}$

A chaque bloc de questions (ou thème) correspond un tableau décrivant les mêmes individus (en ligne) par des variables différentes (en colonne) (18) et contenant (à l'intersection de chaque ligne et de chaque colonne) la réponse d'un éleveur à une question de l'enquête ou une mesure du bilan (figure 1). Les variables quantitatives sont transformées en variables qualitatives après examen attentif de leur distribution. Puis, les tableaux thématiques sont transformés en tableaux disjonctifs où les variables sont remplacées par leurs modalités et les réponses par 0 ou 1 . Les lignes sont équipondérées tandis que l'on associe à chaque colonne un poids égal à $\left(I-I_{k}\right) / I$ (où $I_{k}$ est le nombre d'individus possédant la modalité $k$ et $I$ le nombre d'individus total) dont la somme par tableau est ramenée à l'unité. Ceci vise à équilibrer l'influence de chacun des tableaux.

L'influence d'un groupe de variables sur un autre se mesure à l'aide de la valeur de l'inertie. Ces différences d'inertie s'expliquent non seulement par l'intensité des relations intra-tableaux, mais aussi par le nombre de variables et leur nature (quantitative ou qualitative). En attribuant à chacune des variables un poids inversement proportionnel à la première valeur propre de l'analyse factorielle partielle du groupe auquel elle appartient, l'Afm équilibre le rôle de chacun des thèmes dans l'élaboration de la typologie globale. Concrètement, chaque groupe de variables voit son inertie ramenée à l'unité sans changement de la répartition de l'inertie entre les axes factoriels (7). L'Afm effectue ainsi l'analyse des structures des tableaux qui ont été conservées grâce à la pondération.

L'insigne avantage de l'Afm est donc de raisonner, d'analyser la variabilité intergroupe et intra-groupe par rapport à des ensembles de variables reflétant un mode de fonctionnement, un degré de performance ou de viabilité de l'exploitation (1). L'articulation des thèmes s'interprète à la fois en termes d'analyse du fonctionnement global de l'exploitation et en termes de corrélation entre les thèmes (13). Les analyses et les représentations graphiques ont été réalisées à l'aide du logiciel ADE-4 (29).

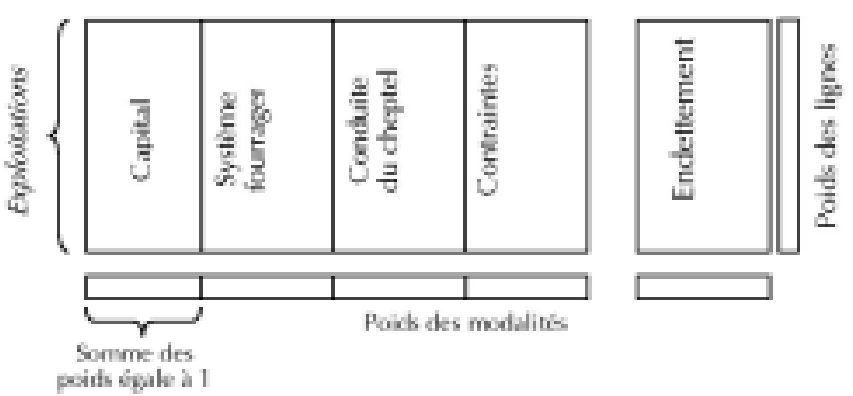

Figure 1 : structure $\mathrm{K}$ tableaux et pondérations associées soumises à l'analyse factorielle multiple. 


\section{RESULTATS}

\section{Analyse interstructure et intra-structure}

L'Afm autorise deux types d'analyse : i) l'analyse interstructure, c'est-à-dire l'analyse des relations ou des corrélations entre groupes de variables (figure 2) ; ii) l'analyse intra-structure, c'està-dire l'analyse des corrélations entre exploitations sur le plan factoriel commun pour l'un ou l'autre des thèmes et, par conséquent, l'appréciation de la variabilité intra-groupe (figure 3).

\section{Analyse interstructure}

L'analyse interstructure est la comparaison globale des tableaux thématiques, c'est-à-dire la réalisation d'une typologie des thèmes. Cette comparaison s'effectue via un système d'axes communs aux groupes de variables. Les coordonnées d'un groupe s'interprètent en termes de liaison globale des variables de ce groupe avec les facteurs communs à l'ensemble des groupes. On peut aussi interpréter les proximités des points en termes de ressemblance entre thèmes.

L'analyse interstructure détermine aussi le plan commun de représentation des variables et des individus, dit «plan moyen ». Ce modèle commun aux différents thèmes est le résultat d'une double optimisation. L'Afm calcule des facteurs qui synthétisent au mieux les corrélations entre toutes les modalités quel que soit leur groupe.

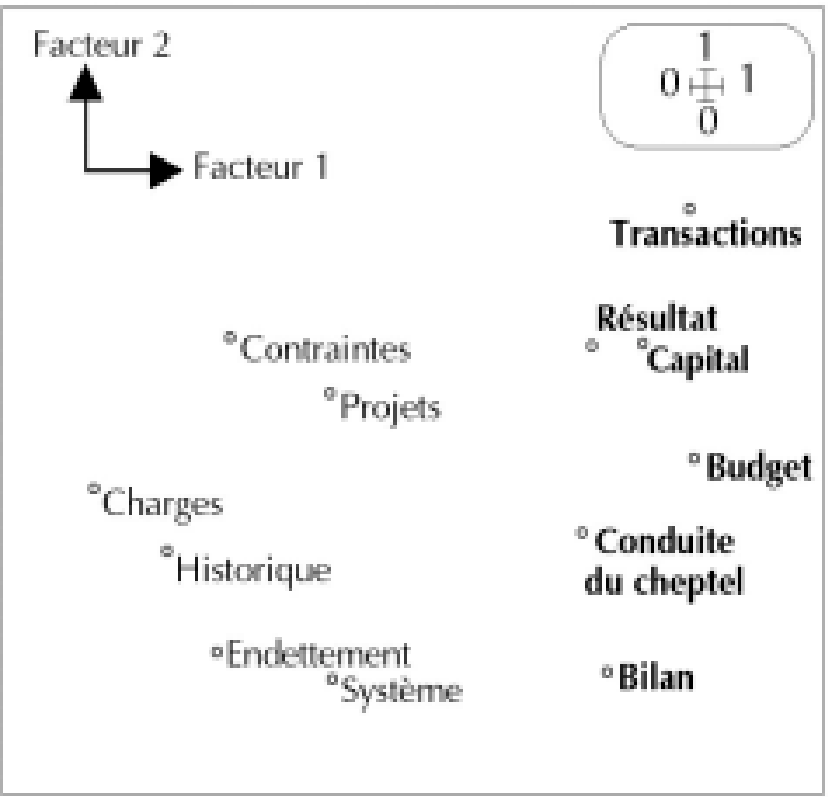

Figure 2 : représentation des thèmes sur le plan 1-2 de l'interstructure de l'analyse factorielle multiple. En gras, les thèmes les plus contributifs aux facteurs communs.

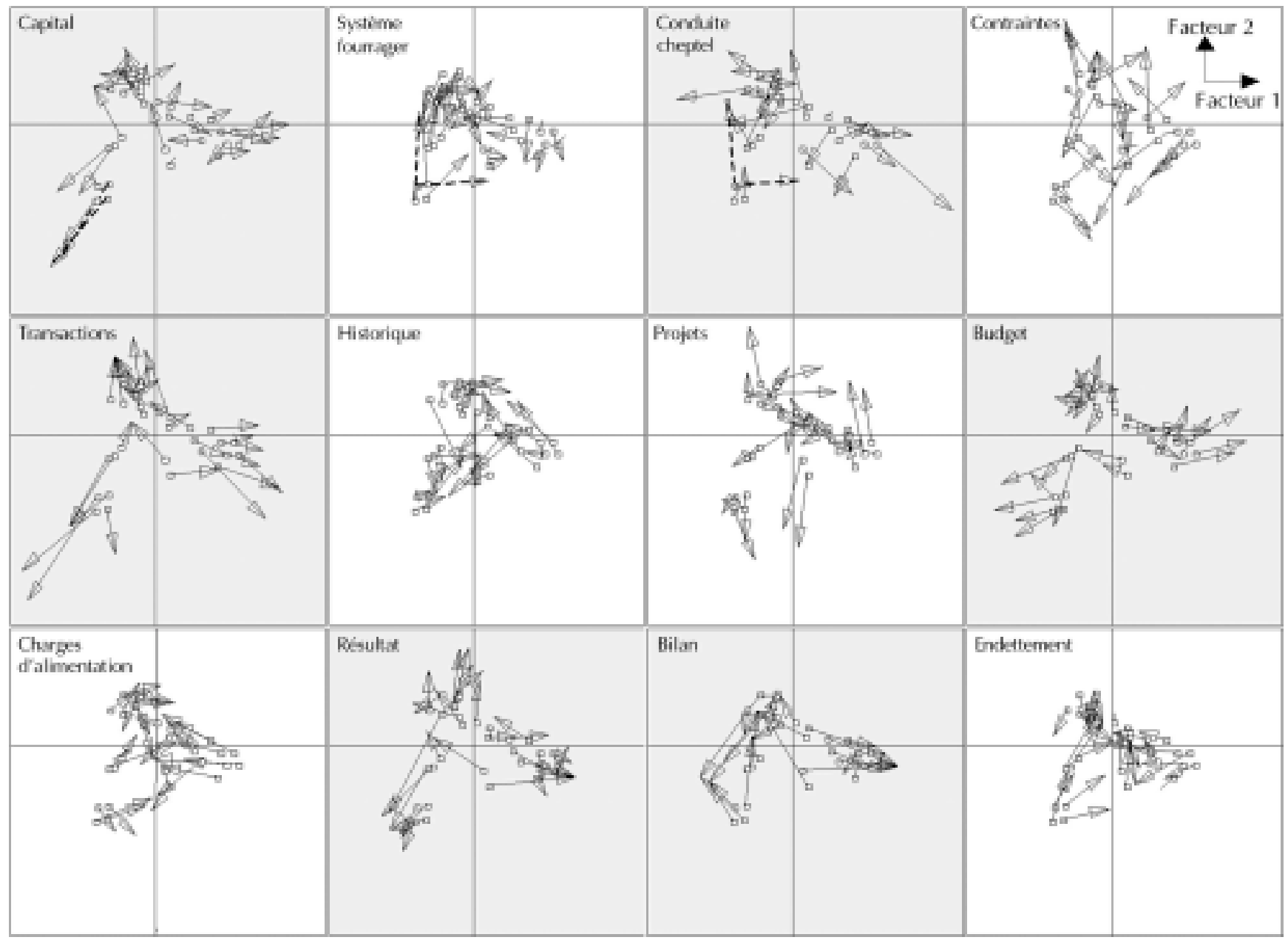

Figure 3 : analyse de l'intra-structure. Représentation des écarts entre élevages moyens et mêmes élevages vus selon les différents thèmes (projection en éléments supplémentaires, extrémité de la flèche) sur le plan factoriel compromis 1-2. En grisé, les thèmes les plus contributifs au plan factoriel commun. 
Ce sont également des axes qui maximisent la variabilité entre les individus. Le plan moyen, à l'instar du plan factoriel d'une analyse factorielle simple, permet par exemple d'identifier les modalités clés de différenciation de la population.

La projection des thèmes a révélé le rôle fortement structurant des thèmes « capital », « transactions » et « résultat » sur le plan moyen $(1 * 2)$, alors que les thèmes relatifs aux « conduites du cheptel », au « bilan » et au « budget » ont structuré l'axe 1 (figure 2). Si les résultats d'exploitation - critère de viabilité économique de l'exploitation - ont davantage été liés au capital vivant et immobilisé de l'exploitation, la conduite du cheptel est apparue fortement conditionnée par le budget, et, donc, la trésorerie disponible et les subventions reçues.

Enfin, la plupart des projets d'exploitation ou projets familiaux se sont élaborés en fonction des contraintes familiales (main d'œuvre disponible) ou environnementales, alors que l'histoire des exploitations ou encore le niveau d'endettement, qui pourraient freiner ou motiver de nouveaux investissements, n'ont pas constitué des facteurs discriminants dans la population étudiée. Ceci s'explique en partie par l'histoire récente du développement laitier à la Réunion, largement inscrite dans les projets publics de réhabilitation des Hauts de l'île. Aussi, pour encourager l'installation d'ateliers laitiers, un système d'aide à l'investissement par le crédit a été accordé à la majorité des exploitations adhérentes à la coopérative.

\section{Analyse intra-structure}

Chaque exploitation pouvait aussi être décrite par des thèmes différents. Aussi une analyse fine des structures communes aux différents thèmes a été réalisée grâce à la projection en éléments supplémentaires des individus partiels (c'est-à-dire les individus vus sous l'angle d'un thème particulier) sur les axes du plan moyen commun (figure 3). L'analyse globale de la figure montre comment les individus s'écartent pour certains thèmes et se rejoignent, en se rapprochant de la moyenne générale, pour d'autres, et elle permet donc de détecter les thèmes les plus structurants ou discriminants de la population. Appelée aussi analyse intra-structure, cette analyse permet de détecter chez les individus proches sur le plan moyen commun les thèmes qui les opposent. Ainsi, il est possible d'identifier les thèmes les plus structurants à l'intérieur d'un groupe d'exploitations, comme de caractériser la variabilité résiduelle à l'intérieur de chaque groupe.

On retrouve, sur le premier plan factoriel commun, le rôle fortement structurant des thèmes liés au capital et aux résultats d'exploitation. La disposition triangulaire des individus permet d'identifier et de caractériser trois pôles : sur l'axe 1, se dessinent deux pôles qui isolent les grandes exploitations laitières de plus de 55 vaches laitières sur au moins 15 ha de cultures fourragères et les petites exploitations de moins de 20 vaches laitières sur moins de 5 ha. On peut parler d'effet de taille entre des structures en groupement agricole d'exploitations en commun (Gaec) qui comptent plus de trois actifs agricoles et les petites structures individuelles qui comptent parfois un seul actif ; l'axe 2 est fortement structuré par des exploitations de taille moyenne, entre 20 et 35 vaches laitières, sur des surfaces de 5 à 15 ha situées principalement dans les Hauts de l'Ouest et la Plaine des Grègues, les Lianes et St Pierre. Les fortes ressemblances sur le plan « historique » et « contraintes » permettent d'identifier un groupe d'anciennes structures qui, soit par expérience soit en raison des difficultés physiques du milieu, ont acquis une gestion prudente de leur exploitation.

On observe aussi que des exploitations voisines au niveau de leur structure (« capital ») ou des résultats économiques (« résultats ») (signalées en pointillés) s'écartent en matière de conduite du cheptel ou de systèmes d'affouragement. Ainsi se dessinent déjà les principales caractéristiques de la diversité des exploitations laitières à la Réunion intergroupes ou intra-groupes.

\section{Typologie des exploitations laitières}

Les coordonnées factorielles des axes moyens obtenus par l'Afm ont été utilisées pour réaliser une classification ascendante hiérarchique $(\mathrm{CAH})$ qui permet de ne retenir que l'essentiel de l'information contenue dans les tableaux pour discriminer les exploitations. Cette $\mathrm{CAH}$ permet de définir des groupes d'exploitations laitières les plus homogènes possible. La typologie réalisée sur les sept premiers axes factoriels permet de faire émerger des groupes très proches sur le plan factoriel moyen 1-2 qui synthétisent les ressemblances et les oppositions entre six types d'exploitations laitières différentes.

La projection des classes d'éleveurs laitiers sur le premier plan montre une première opposition entre les classes 2 et 3 et les quatre autres classes sur l'axe factoriel 1 (figure 4). On distingue les grandes et moyennes structures des plus petites qui se différencient aussi en termes de budget et de résultats du bilan. Les quatre groupes qui représentent les plus petites exploitations se différencient sur l'axe 2, facteur de l'intensité et des types de transactions, qui pourrait aussi s'appeler l'axe des stratégies économiques alimentaires en situation de pression foncière, que cette pression provienne de l'exiguité des parcelles ou des contraintes physiques du milieu.

\section{Type 2 (Egaec). Grandes exploitations basées sur un système mixte fourrage-pâturage}

Le type 2 représente les grandes exploitations ayant le plus important cheptel laitier (plus de 55 vaches présentes), sur plus de 35 ha de terres dont au moins un tiers en pâture. La plupart de ces exploitations, qu'elles soient en Gaec ou de statut individuel, ont connu une croissance importante depuis les années 1990. Situées dans les Hauts, les prairies sont généralement des prairies de graminées tempérées dont plus de 15 ha sont récoltés sous forme d'ensilage balle ronde. Mais ces éleveurs pratiquent aussi une forte complémentation en concentré : plus de $13 \mathrm{~kg}$ par vache laitière par jour pour des rendements de 10 à 15000 l/ha. Cependant, il s'agit de la consommation totale de concentré et nombre de ces exploitations se diversifient aujourd'hui par l'engraissement de jeunes taurillons et le développement d'un atelier génisse.

Ces exploitants ont bâti leur capital foncier, cheptel et matériel sur plus de vingt ans. Aujourd'hui, ils possèdent un équipement presque complet : chaîne d'ensilage en balles rondes enrubannées, salle de traite, voire hangar à foin et nouveau bâtiment pour un atelier génisse. Cependant, si les prêts de moyen et long termes pour les gros investissements sont en partie remboursés, ces exploitations sont obligées de recourir à de nombreux emprunts de court terme pour le fonctionnement de leur exploitation et notamment pour assurer les charges de structure qui représentent près de 42 p. 100 des charges d'exploitation. Si ce groupe présente les meilleurs résultats d'exploitation en termes de chiffre d'affaires et d'excédent brut d'exploitation, les indicateurs de rentabilité et de solidité restent fragiles en raison, d'une part, des fortes charges de structure et, d'autre part, des emprunts de court terme. Des gains pourraient être réalisés par le biais d'un meilleur rationnement, notamment par un recours plus raisonné aux concentrés, mais aussi par le biais de contrats de travail ou d'associations plus appropriés pour les exploitations hors Gaec.

Ces exploitations font office aujourd'hui de modèles pour l'élevage laitier à la Réunion et la plupart des éleveurs poursuivent 


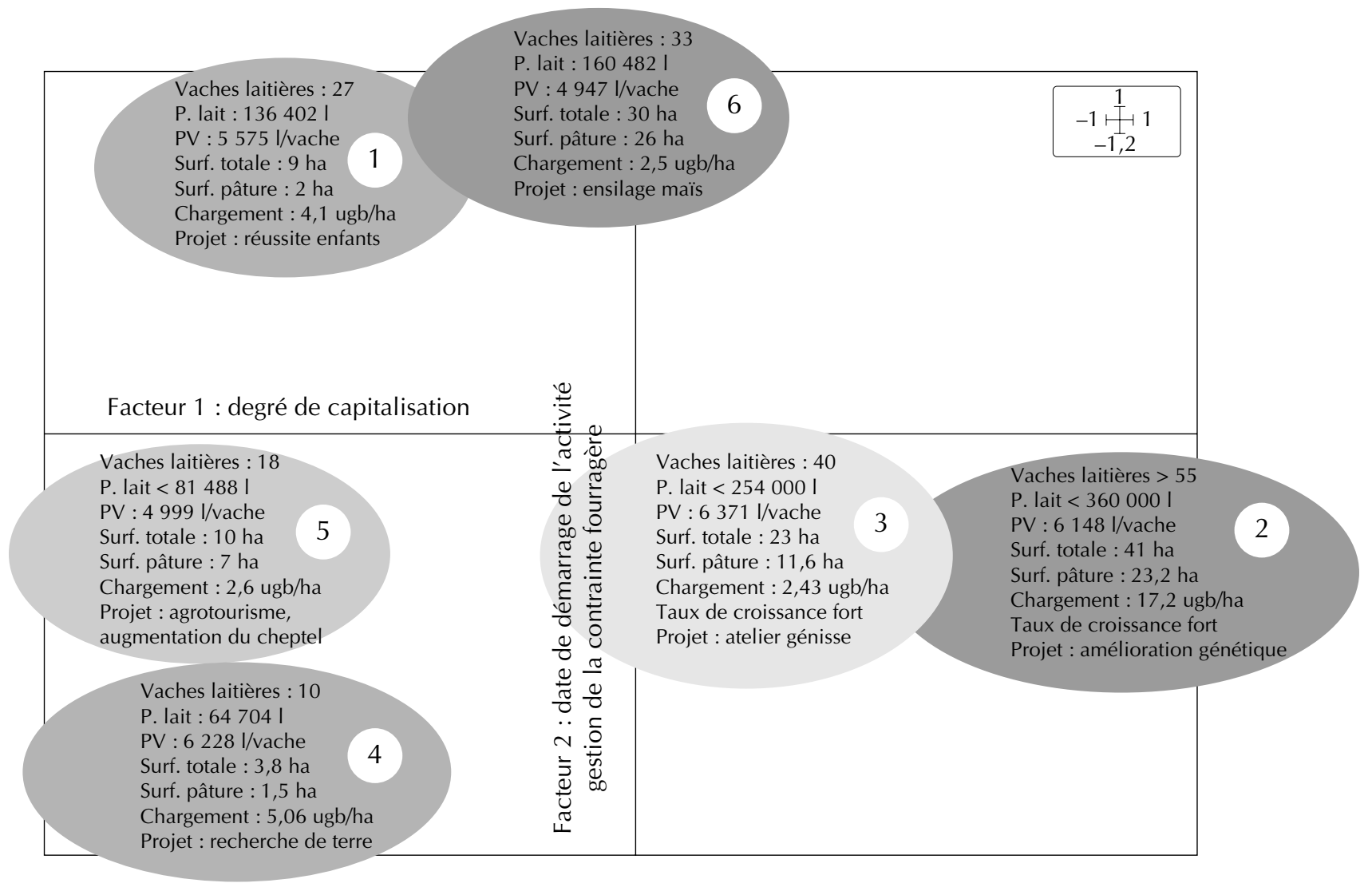

Figure 4 : groupes de la typologie des exploitations laitières sur le plan factoriel commun 1-2 de l'analyse factorielle multiple.

toujours des objectifs d'amélioration de la productivité laitière par l'importation de génisses et aussi par l'investissement dans des semences de taureaux améliorateurs.

\section{Type 3 (Epdl). Exploitations de type Plan de développement laitier ayant une bonne autonomie fourragère}

Le type 3 comprend les exploitations intermédiaires entre les grosses structures et les plus petites. Ces exploitations comptent un troupeau moyen de 35 à 55 vaches laitières sur 15 à 35 ha de prairies à dominante graminées tempérées. Ces exploitations laitières relativement récentes se sont constituées après 1990 avec l'aide d'un plan de développement laitier. Bien qu'autonomes en matière d'affouragement (vente d'un surplus fourrager à l'extérieur), ces exploitations recourent à des quantités importantes de concentrés : $10-13 \mathrm{~kg}$ de concentrés/vache/j (soit entre 6 et $7000 \mathrm{~kg}$ de concentrés/vache/an) pour atteindre leur objectif de production laitière, ce qui explique en partie le niveau élevé des charges opérationnelles (plus de $53400 €$ par an). Si ces exploitations sont autonomes du point de vue fourrager par rapport à leur besoin, on ne peut parler d'autonomie alimentaire. Cette stratégie d'alimentation peut s'expliquer en raison du faible coût des concentrés à la Réunion et du fort taux de rémunération du fourrage à la vente.

A la différence du type 2, les exploitations du type 3 semblent mieux maîtriser leur charge de structure qui représente moins d'un tiers des charges d'exploitation. Ceci est essentiellement lié au non-recours à des salariés permanents. Ces exploitations en fin de Pdl sont dans une phase de remboursement des prêts de moyen et long termes et préfèrent donc recourir à des stagiaires ou ouvriers saisonniers pour éviter d'alourdir les charges de structure. Malgré une autonomie fourragère relativement élevée, ces exploitations enregistrent d'importantes charges opérationnelles. Le détail des charges opérationnelles révèle la part non négligeable des frais d'élevage et des fournitures diverses qui s'explique en partie par les caractéristiques des chefs de ménage. En effet, il s'agit pour beaucoup d'entre eux de jeunes qui ont reçu une formation professionnelle en élevage et sont soucieux de cadrer avec leur enseignement. L'expérience et l'assurance progressive dans leur appareil de production permettront progressivement de réduire les frais superflus. Dans les charges de structure, on note la part importante des charges locatives relatives au foncier ; en effet, ces jeunes se sont installés sur des terres en location ou fermage, entraînant des coûts actuels importants.

Ainsi ces exploitations entament aujourd'hui une phase de consolidation (maintien du troupeau en l'état) qui passe par le remboursement progressif des emprunts de long terme. Leur souhait à terme est d'alléger leur charge de travail par l'embauche d'un ouvrier, mais aussi de se diversifier par l'investissement dans l'immobilier ou le foncier. Ces projets soulignent le caractère spéculatif que revêt l'activité laitière dans leur exploitation.

\section{Type 6 (Epat). Exploitations de type « extensif » sur pâture}

Les exploitations de type 6 sont très proches de celles de type 1 sur le plan factoriel commun, mais elles se distinguent par le système fourrager. Elles se distinguent également des autres groupes de par une alimentation sur pâture de Kikuyu, système qui domine dans l'élevage laitier des Hauts de l'Ouest. Les chefs d'exploitation, fils de planteurs de canne, se sont installés dans les Hauts avant 1990, largement incités par le développement des politiques d'aide à l'installation des années 1980 et début 1990. Ces exploitations enregistrent de faibles performances laitières, liées aux problèmes récurrents d'affouragement : moins de 5000 1/vache/an avec près de $10-13 \mathrm{~kg}$ de concentrés/vache laitière/j. Elles ont recours de préférence au foin de Chloris ou résidus de canne presque toute l'année, ce qui représente avec les concentrés près de 64 p. 100 des charges variables. 
Ainsi, pour ces exploitations, le choix de la conduite alimentaire sur pâturage de Kikuyu résulte davantage des conditions physiques et climatiques de la zone que d'une stratégie extensive de conduite : la plupart des terres sont difficilement mécanisables et l'intensité des périodes de sécheresse ne permet guère l'implantation de graminées telles que le dactyle ou le Ray-grass. En revanche, ces exploitations souhaitent développer ou développent des parcelles situées plus dans les Bas pour faire du maïs ensilage en silos taupinières.

\section{Type 1 (Efauch). Exploitations ayant une gestion économique prudente}

Le type 1 regroupe des structures d'exploitation relativement anciennes (installation avant 1985), situées majoritairement dans la Plaine des Grègues ou St Joseph. Ces éleveurs ont le plus souven hérité de l'exploitation de leur père. Elles sont dotées d'un troupeau de 20 à 35 vaches laitières sur 9 ha en moyenne, soit un chargement moyen de $4,1 \mathrm{ugb}^{2} / \mathrm{ha}^{*}$. Malgré cette forte contrainte foncière, ces exploitations recourent modérément aux concentrés : moins de $10 \mathrm{~kg} / \mathrm{vache} / \mathrm{j}$, tout en maintenant de meilleures performances que le type 6 (soit 5 000-6 000 l/vache/an). Cette gestion prudente des charges de complémentation explique en partie le très bon taux de rentabilité brute d'exploitation enregistré (plus de 45 p. 100).

Le mode d'affouragement est essentiellement basé sur la fauche en vert quotidienne. Ce système d'affouragement est bien sûr lié aux contraintes régionales, mais aussi au morcellement du parcellaire qui rend difficile tout projet de mécanisation, excepté quelques exploitations qui pratiquent de l'ensilage de balles rondes enrubannées de Chloris et parfois de Brome dans des zones plus propices.

Il faut noter le faible niveau d'endettement (moins de 25 p. 100), qui allège les charges financières. Ainsi, à la différence de l'ensemble des autres types, le type 1 se caractérise par une situation économique et financière très stable, grâce à une gestion prudente, et ce, malgré une faible autonomie fourragère.

\section{Type 4 (Ehors). Exploitations de type hors sol}

Ce type regroupe les exploitations comptant un troupeau de moins de 20 vaches laitières sur moins de 5 ha. Il s'agit d'un système quasiment hors sol qui se développe depuis à peine cinq ans, en raison de la forte pression foncière dans les bassins laitiers, notamment celui de la plaine des Cafres. De fait, ces exploitations sont très fortement dépendantes de l'extérieur pour leur affouragement. Dans la plupart d'entre elles, sont distribués plus de $13 \mathrm{~kg}$ de concentré/vache/j. Il en résulte, du point de vue économique et financier, de fortes charges d'alimentation qui s'élèvent à plus de $1300 €$ par vache. L'achat des concentrés représente entre 35 et 41 p. 100 du prix du lait. Ces exploitations connaissent aussi une forte pression financière à court terme en raison du recours fréquent aux emprunts et à la quasi-absence de fonds de roulement.

Pour l'instant, le principal objectif de ce groupe est bien sûr l'assurance d'un revenu pour la famille dans un contexte de fortes contraintes de trésorerie à court terme. Il faut noter que certains chefs d'exploitation de ce groupe se consacrent à l'élevage laitier depuis moins de cinq ans, après avoir pratiqué d'autres activités (parfois non agricoles). Dès lors, ils ont le souci de former rapidement un capital suffisant pour assurer la viabilité de leur exploitation. Les autres sont plus orientés vers la cession de leur activité à leurs enfants et investissent en vue de la succession.

\section{Type 5 (Ejeun). Jeunes exploitations en cours de constitution}

Le type 5 rassemble les exploitations en phase de constitution de leur cheptel dont l'objectif est d'agrandir le troupeau de plus de

* Unité de gros bétail par hectare
50 p. 100. A l'instar du type 4, ces exploitations connaissent une forte contrainte foncière (moins de 5 ha de surfaces totales) qui est maîtrisée du point de vue économique par le choix d'un système peu productif : les charges d'alimentation représentent moins du tiers des charges totales pour une productivité inférieure à 45001 par vache. Les apports fourragers de l'exploitation sont assurés par une fauche quotidienne en vert.

Si les exploitations des types 4 et 5 ont des structures voisines (petit cheptel, forte contrainte foncière et d'affouragement), elles se distinguent par le mode de financement de l'activité. A la différence du type 4 , les exploitations du type 5 réduisent au minimum l'utilisation de concentrés, préférant axer leur effort sur la valorisation de leur foncier. En outre, ces nouvelles exploitations en voie de constitution bénéficient de prêts et d'aide à l'installation de moyen et long termes, ce qui allège la contrainte de trésorerie à court terme. Par rapport au type précédent, le type 5 se caractérise par une gestion plus prudente de la trésorerie.

Il s'agit de jeunes exploitants localisés en zone périurbaine (zone du Tampon, à proximité de St Pierre, pour la plupart) qui ont développé cette activité avec un souhait de se diversifier vers l'agrotourisme. Ce groupe se situe donc à l'opposé des exploitations spécialisées dont l'objectif est d'accroître toujours plus leur rendement laitier par vache. En outre, il leur est difficile aujourd'hui d'accroître leur cheptel sans terres supplémentaires. Les souhaits d'investissement à court terme portent davantage sur l'équipement de la salle de traite et les bâtiments.

Les types 4 et 5 se distinguent des autres types par le faible niveau de soutien extérieur, essentiellement lié à la taille de l'exploitation, puisque la majorité des primes et des subventions annuelles sont fonction, soit de la surface, soit du nombre d'ugb, soit d'une combinaison des deux (indemnité spécial montagne, détaxe gasoil, primes à la conservation des génisses, etc.). On est surpris aussi de noter la faible part des charges d'alimentation dans les charges totales pour deux types d'exploitation qui connaissent d'importants problèmes d'affouragement en raison de la modicité des parcelles. Le détail des charges opérationnelles montre d'importantes charges vétérinaires dont le montant peut être proche de celui des plus grosses structures pour un cheptel trois à quatre fois moindre. Dès lors, un des problèmes majeurs de ces exploitations est la maîtrise des soins sanitaires dans le cadre d'un modèle fortement intensif, peut être plus propice aussi aux maladies.

L'histoire des exploitations à la Réunion comme la caractérisation des groupes permet d'ébaucher des hypothèses d'évolution pour certains types d'exploitation (figure 5). Ainsi, le type 5 des petites exploitations (Ejeune) se trouve à l'intersection entre le groupe des petites exploitations en difficulté, des moyennes exploitations de 25-30 vaches laitières ayant acquis une bonne stabilité et solidité financière, et des moyennes exploitations de 40 vaches laitières ayant poursuivi leur investissement grâce aux Pdl. Il est vrai que le cheminement du type 5 vers les moyennes exploitations est fortement dépendant de la question foncière. Sous contrainte de faible disponibilité foncière, les exploitations de ce groupe peuvent soit s'acheminer vers le type 1 grâce au maintien des aides à l'installation et à la capitalisation, soit stagner et se retrouver dans le type 4 au bout de quelques années.

L'analyse des groupes donne une première approche de la diversité des exploitations à la Réunion en fonction de leur date d'installation, de leur taille et de leurs modes de gestion de l'alimentation qui se distinguent de la gestion du système fourrager. Elle permet d'élaborer un premier diagnostic sur les atouts et les faiblesses de chaque type d'exploitation. De l'analyse statique se dégagent aussi différentes voies ou modes d'évolution des exploitations laitières qui pourraient se décliner en deux principaux modes : changement 


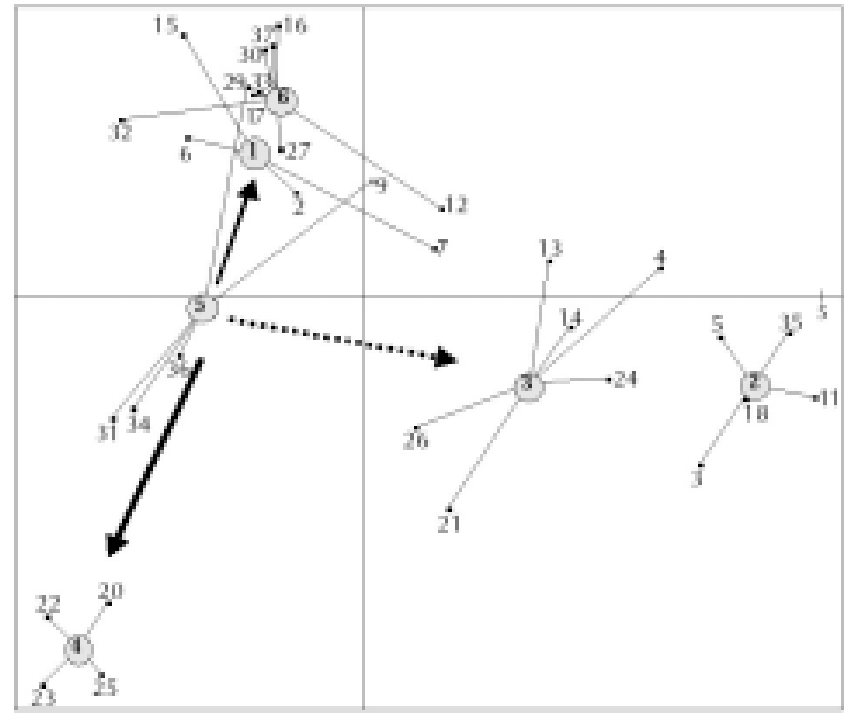

Figure 5 : hypothèses d'évolution des types d'exploitation. Représentation des centres de gravité des groupes de la typologie élevages laitiers et connexion des élevages d'un même groupe à l'aide d'un segment, sur le plan factoriel commun 1-2 de l'analyse factorielle multiple.

structurel lié à la capitalisation ou décapitalisation - largement induit par les politiques de soutien à l'élevage bovin comme les $\mathrm{Pdl}$ - et adaptation par les facteurs liée en partie à un changement des modes d'alimentation (19).

\section{Changements structurels ou adaptation}

Pour approfondir ces hypothèses d'évolution par changement de structure ou d'adaptation, les groupes d'exploitation ont été comparés selon le degré d'intensification (coût de production/ugb) et les changements structurels (nombre d'ugb/actif) (figure 6). Si le groupe des grandes exploitations (Etgaec) a tendance à augmenter le nombre d'ugb par actif (changement structurel) alors que les exploitations de type Pdl (Epdl) tentent davantage de s'intensifier, ces deux groupes sont situés aux frontières des axes et ont bien des conduites combinées. Seul le groupe des petites exploitations de type hors sol (Ehors) joue la carte de l'intensification. Pour ce groupe, l'intensification semble un passage obligé alors qu'il s'agit plus d'un choix ou modèle de conduite pour les exploitations de type Pdl (Epdl). Les exploitations de la plaine des Grègues et de St Joseph ont tendance à intervenir plus sur la structure alors que les deux derniers groupes (Epat et Ejeun) sont dans une situation incertaine qui rend difficile tout processus d'intensification ou de changement structurel.

Les nuages de points des exploitations reflétant la structure des charges selon la taille de l'exploitation (exprimée en nombre d'ugb) montrent une certaine homogénéité des coûts variables (entre 750-1 $500 € /$ ugb) pour les exploitations de taille moyenne (entre 30 et 40 ugb), avec de fortes disparités au-delà et en deçà (figure 7). Près de la moitié des petites exploitations de moins de $20 \mathrm{ugb}$ et des grandes exploitations de plus de $40 \mathrm{ugb}$ ont des charges variables supérieures à $1500 €$ par ugb. Il s'agit essentiellement des plus petites exploitations qui comptent à peine $15 \mathrm{ugb}$ ou des exploitations de 50 à 60 ugb. Si les premières souffrent incontestablement d'un problème de forte dépendance au marché pour l'alimentation du bétail, il s'agirait pour les moyennes d'un problème de maîtrise des frais d'élevage et des petites fournitures d'entretien. En revanche, ce sont essentiellement les moyennes structures (entre 30 et $40 \mathrm{ugb}$ ) qui enregistrent les plus gros écarts en matière de charges structurelles (figure 8). Ce sont ces mêmes

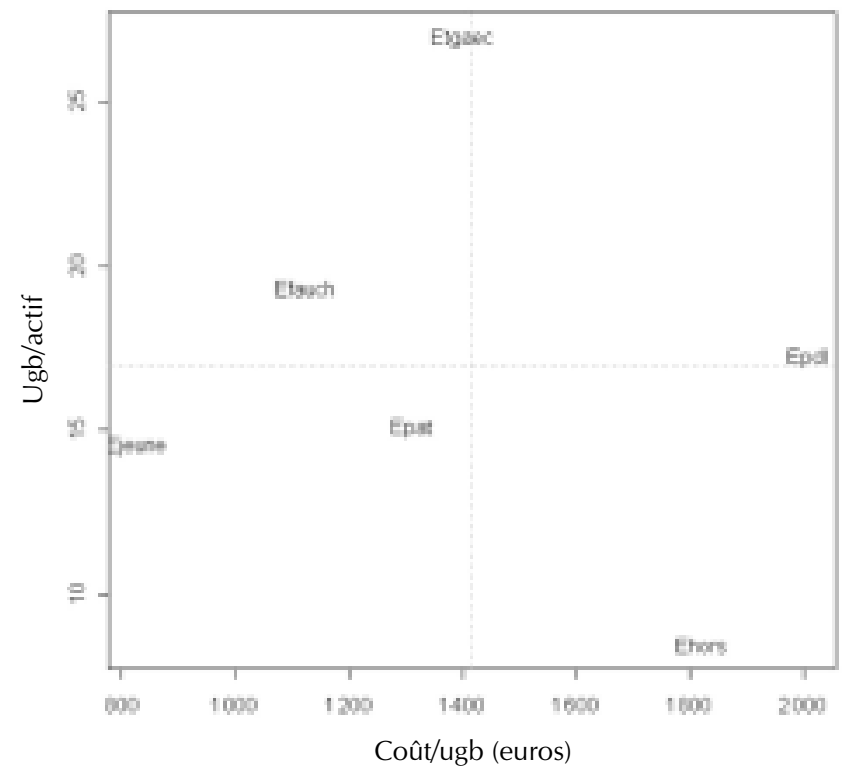

Figure 6 : représentation du nombre d'ugb par actif en fonction des coûts de production par ugb pour les types $d^{\prime}$ exploitations laitières $($ Egaec $=$ grandes expl. basées sur système mixte fourrage-pâturage ; Epdl = expl. type Plan de développement laitier avec bonne autonomie fourragère ; Epat $=$ expl. type extensif sur pâture ; Efauch $=$ expl. avec gestion économique prudente ; Ehors = expl. type hors sol ; Ejeun $=$ jeunes expl. en cours de constitution). Représentation du point moyen en trait pointillé.

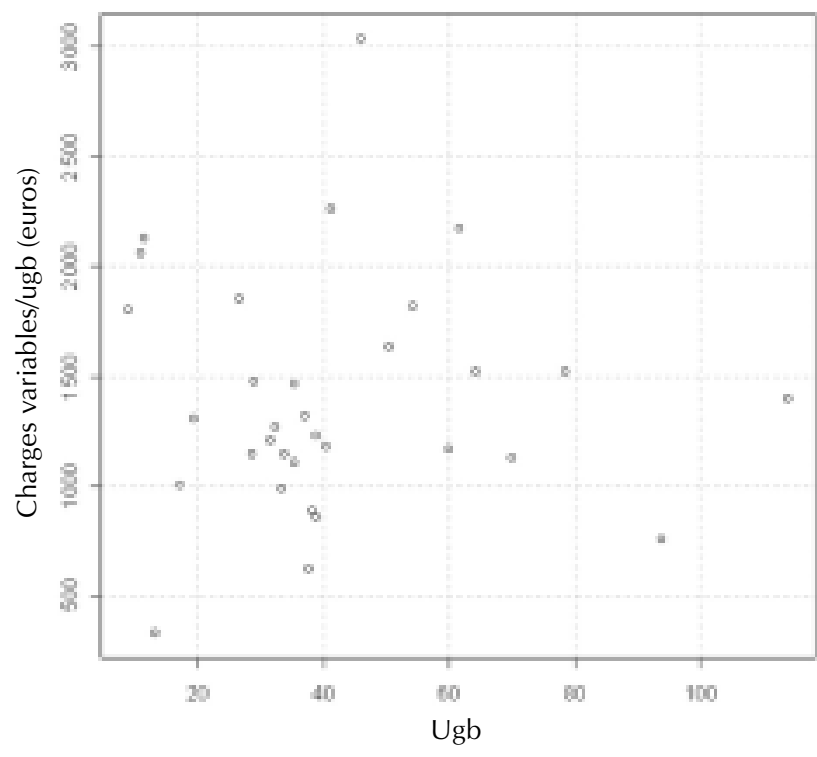

Figure 7 : représentation des charges variables par ugb en fonction du nombre d'ugb par exploitation.

exploitations qui enregistrent des charges moyennes en main d'œuvre de $18320 €$ (contre $6870 €$ pour les exploitations qui enregistrent des charges de structure de moins de $610 € / \mathrm{ugb}$ ). Ainsi, il est possible de distinguer deux seuils difficiles dans l'évolution des exploitations : i) le changement structurel relatif au travail pour le passage à plus de 40 ugb pour les exploitations de moins de 2 UTA (actif agricole) ; ii) la gestion de l'alimentation pour les petites et grandes unités, liée respectivement à la gestion de la pénurie pour les petites exploitations et la maîtrise de l'alimentation pour les plus grandes. 


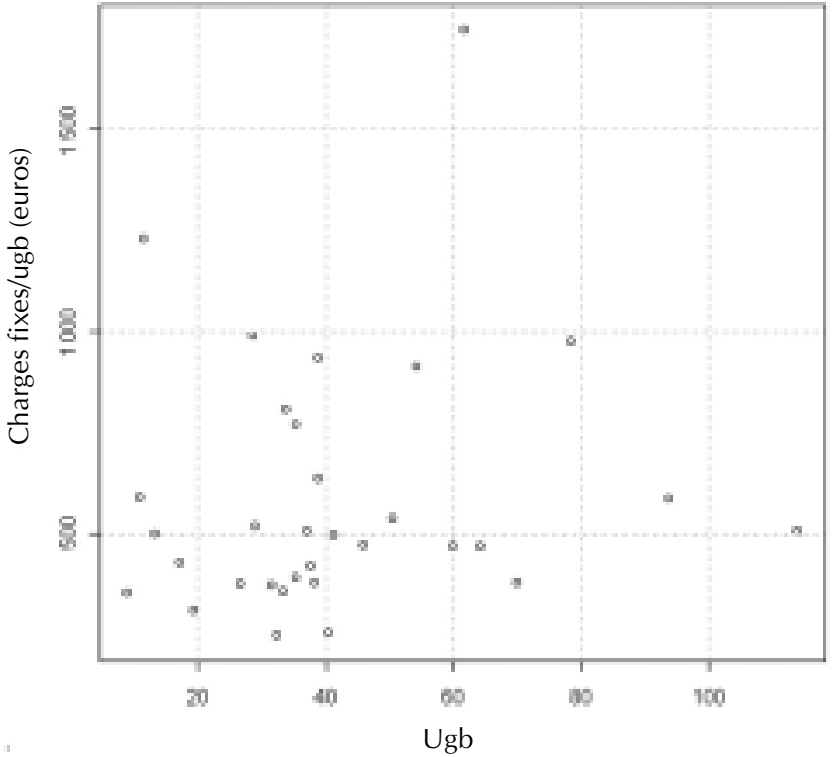

Figure 8 : représentation des charges fixes par ugb en fonction du nombre d'ugb par exploitation.

La représentation des courbes moyennes - obtenues par régression locale (6) - des coûts de production, des produits d'exploitation et du chiffre d'affaires (produit + primes et subventions) en fonction de la taille de l'exploitation montre une faible marge bénéficiaire pour l'ensemble des éleveurs et donc un faible avantage lié à la taille ou aux conditions agro-climatiques et structurelles des exploitations (figure 9). La principale marge proviendrait des primes et subventions à la production ou à l'hectare pour les moyennes et grandes exploitations. Plusieurs facteurs peuvent expliquer ce phénomène : i) tout d'abord le rôle pilote de la coopérative dont l'un des soucis est d'augmenter la production laitière en soutenant autant l'installation des jeunes et des petites structures que les efforts d'investissement des grandes structures ; ii) le rôle de conseil de l'ensemble des partenaires qui permet d'éviter certains écueils et gérer l'ensemble des aides; iii) la gestion prudente de l'ensemble des éleveurs dans un milieu physique soumis à de nombreuses perturbations (cyclones, sécheresse, etc.). On pourrait dès lors se demander pourquoi les éleveurs s'acheminent ou souhaitent s'acheminer vers des structures plus importantes. Outre des considérations

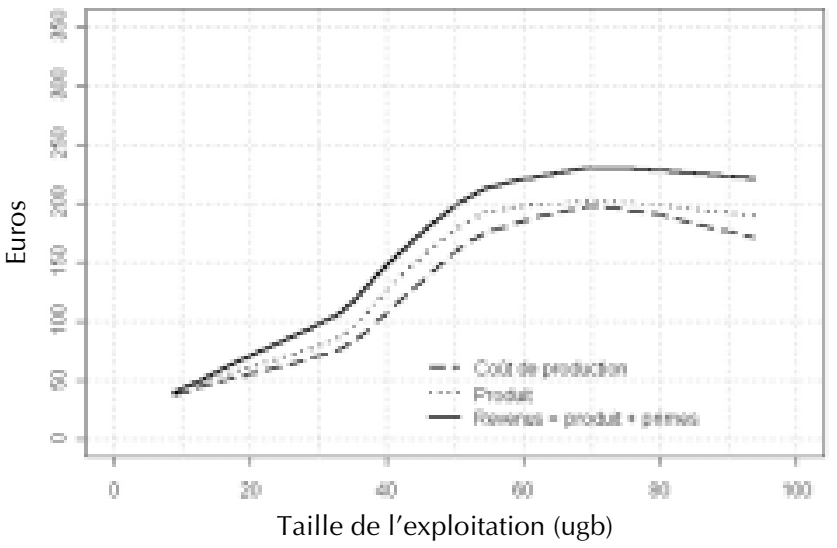

Figure 9 : représentation des courbes moyennes des revenus (produits + primes), du produit et des coûts de production de l'exploitation (en milliers d'euros) en fonction de sa taille (en ugb), obtenues par régression locale. de prestige dans un cadre aussi innovant que l'élevage laitier à la Réunion, l'élevage constitue toujours une source d'emploi pour la famille, puis pour les enfants en âge de travailler. Cependant, le faible nombre d'exploitations de plus de 60 ugb rend difficile l'élaboration d'un diagnostic fiable bien que ce soient ces mêmes exploitations qui font office de modèles pour les autres.

La faible taille de l'échantillon ne permet pas de reproduire les courbes moyennes par groupe. En revanche, les résultats moyens par groupe montrent que les primes représentent près de 50-60 p. 100 de la marge de chaque classe, à l'exception du type 4 (moins de 20 p. 100) et du type 6 (plus de 90 p. 100). Le type 4 des petites exploitations en stagnation, voire régression, bénéficie de peu d'aide à la production en raison du faible niveau de capitalisation, à l'inverse du type 6 qui bénéficie de l'ensemble des aides en raison de son isolement géographique et des difficultés liées au milieu physique.

\section{DISCUSSION}

Si cette structuration de la population étudiée peut paraître peu originale à première vue (les grandes structures enregistrent les flux monétaires les plus importants, les plus anciennes structures en voie de cession ont une gestion plus prudente ou modérée, etc.), elle fait apparaître le rôle plus secondaire des charges d'alimentation et du système fourrager dans la constitution des groupes. Or, les modes d'affouragement (combinaison des systèmes fourragers, du recours aux concentrés et du degré d'autonomie fourragère) sont bien souvent cités comme facteurs contraignants de l'élevage laitier à la Réunion et expliqueraient l'importance des charges opérationnelles et, par voie de conséquence, les résultats d'exploitation différenciés. Plusieurs hypothèses peuvent être émises :

- les thèmes relatifs aux systèmes fourragers, aux contraintes géographiques et au degré d'autonomie fourragère seraient partagés par l'ensemble de la population. Pourtant, les microclimats réunionnais ont été bien souvent analysés comme facteurs de différenciation des systèmes et de difficultés différenciées pour l'élevage ;

- on pourrait également penser à une quasi-individualisation des problèmes fourragers en fonction de la contrainte foncière, du cheptel et de la zone qui rend difficile toute comparaison ou regroupement ;

- ces thèmes peuvent aussi participer à une typologie de la population étudiée indépendante de la précédente ;

- il peut s'agir aussi d'un problème de codification des informations relevées au cours de l'enquête ou du choix des variables pour approcher le système fourrager.

$\mathrm{Au}$ final, l'absence de toute implication de ces thèmes peut amener à rediscuter des questions formulées dans le questionnaire pour mieux les approcher.

Une nouvelle Afm a été réalisée sur des tableaux de données mixtes $(11,15)$, en évitant le recours à la codification pour les données quantitatives. Les blocs de variables quantitatives et qualitatives ont fait l'objet d'une analyse préliminaire séparée, respectivement une analyse en composantes principales (Acp) et une analyse des correspondances multiples $(\mathrm{Acm})$. La représentation des thèmes ${ }^{1}$ sur le plan de l'interstructure de l'Afm sur le tableau mixte a conduit les auteurs à écarter l'hypothèse de problèmes liés à la codification (figure 10), l'interstructure étant en effet sensiblement la même que celle de l'analyse sur variables qualitatives (figure 3 ).

\footnotetext{
${ }^{1}$ La plupart des variables du thème « contraintes » étaient très liées au mode de gestion des fourrages. Elles ont été intégrées au thème « système fourrager » du tableau mixte qui ne comporte plus que 11 thèmes
} 


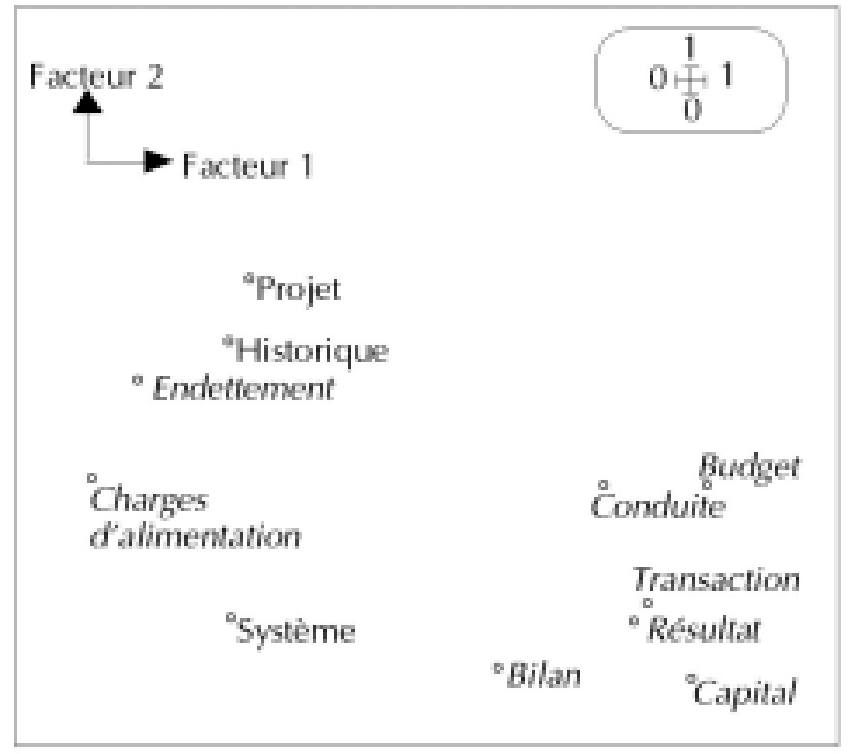

Figure 10 : représentation des thèmes sur le plan 1-2 de I'interstructure de l'analyse factorielle multiple sur tableau mixte. En italique, les thèmes avec variables quantitatives.

La projection sur le plan moyen de l'analyse initiale des centres de gravité de la partition obtenue après une classification des élevages sur le seul thème «système fourrager » révèle l'existence d'une forte variabilité intra-groupe (figures 5 et 11). Ces résultats montrent le rôle peu structurant de ce thème dans la construction d'une typologie globale et conforteraient l'hypothèse d'un fort particularisme des exploitations sur le plan du système fourrager qui expliquerait le rôle secondaire de ce thème dans l'analyse.

Cependant, l'expérience montre aujourd'hui que les groupes observés dans la typologie précédente selon des critères autres que le système fourrager ont été identifiés par les techniciens de la

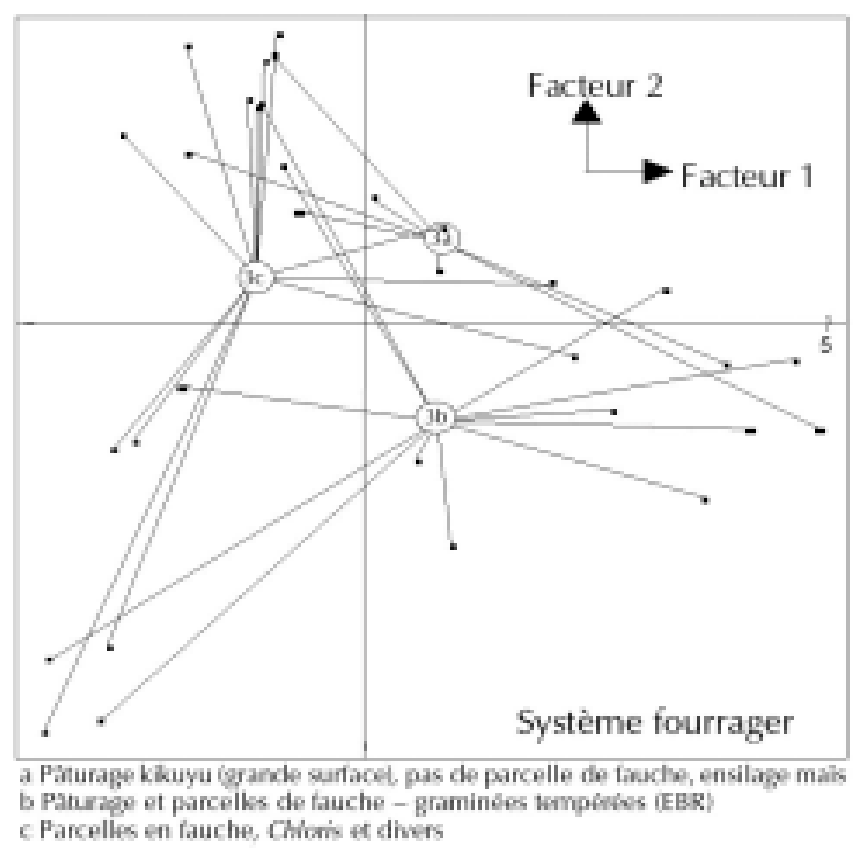

Figure 11 : représentation des centres de gravité de la partition obtenue après une classification des élevages sur le thème "systèmes fourrager " sur le plan 1-2 moyen de l'analyse factorielle multiple sur tableau qualitatif. coopérative en partie par la zone et le degré d'autonomie alimentaire. Pour expliquer ce paradoxe apparent, il faut revenir aux choix des variables dans chaque groupe. En fait l'ensemble des exploitations laitières de la Réunion, quelles que soient leur situation géographique, qui explique en partie le système fourrager dominant, recourent aux achats d'aliments extérieurs et aux concentrés, qui figurent dans le thème " transaction », et le degré d'autonomie est davantage lié au capital foncier par rapport au capital vivant qu'au type de graminées ou au mode de collecte. Si le système de culture fourrager est fortement conditionné par la situation géographique de l'exploitation et relèverait d'un fort particularisme, tous les exploitants recourent à une forte quantité de concentrés et l'autonomie fourragère est largement évaluée à la Réunion en termes de volume de transaction monétaire : achat ou vente par rapport à un niveau d'entretien minimal des vaches. Enfin, le système fourrager proprement dit est généralement un système complexe, fonction du système élevage, de mécanisation, des choix des producteurs, des contraintes de travail et de trésorerie, etc., et dans ce sens nécessiterait peut être une autre typologie.

Ainsi, la combinaison de méthode à dire d'expert par construction préalable des hypothèses de regroupement des exploitations et l'analyse multivariée, qui garantit une certaine exhaustivité et objectivité par rapport aux facteurs structurants de regroupement, a permis aux auteurs à la fois de comprendre la diversité des exploitations et d'approcher la complexité des exploitations à l'intérieur des groupes. La construction de l'objet modélisé par itération, alternant le recueil d'informations auprès des partenaires et l'analyse successive de deux échantillons d'exploitation, a permis à la fois d'éviter le choix arbitraire des variables et de se prémunir du risque de vision potentiellement simplificatrice ou subjective des techniciens. L'analyse intra-structure a également permis d'élaborer, à partir des écarts des exploitations par rapport à leur position moyenne, qui elles définissent les pôles, des critères de ressemblances, à l'instar de l'analyse à dire d'expert, et ce, sur la base de la définition d'une clé typologique. Cette clé pourrait à court terme conduire à la réalisation d'une typologie sur l'ensemble des élevages laitiers de l'île.

L'analyse de groupes conduite en termes de changements structurels ou d'intensification souligne le caractère tout à fait original du développement des exploitations laitières à la Réunion par rapport aux élevages des zones traditionnelles de montagne. Il est vrai qu'en France métropolitaine, il est rare de trouver des petites exploitations entièrement hors sol en zone de montagne. Ce type d'exploitation s'écarte des moyennes et grandes exploitations qui connaissent un développement à peu près régulier de leur structure et du degré d'intensification. En outre, les courbes de tendance semblent montrer soit une faible marge bénéficiaire des élevages en fonction de la taille soit une très bonne répartition des aides et des primes. Dans tous les cas, les primes et les aides apparaissent comme des éléments structurels indispensables au développement de l'élevage laitier à la Réunion et reposent dans des termes plus précis la question de la viabilité et de la durabilité des différents systèmes d'exploitation dans un contexte institutionnel changeant au Nord comme au Sud. Ces premiers éléments issus de l'analyse typologique feront l'objet d'analyses plus approfondies dans le cadre d'un modèle de programmation mathématique, afin de tenir compte de la dynamique complexe des systèmes étudiés.

\section{CONCLUSION}

Cette première analyse par l'Afm a permis d'approcher la complexité et la diversité des exploitations réunionnaises au travers des analyses interstructure et intra-structure de la population étudiée. Des facteurs communs de différenciation des élevages ont été mis 
en évidence de façon optimale, pour apprécier les divergences ou ressemblances entre thèmes qui caractérisaient un même élevage. Ainsi ont pu être distingués six types d'exploitation relativement stables du point de vue technique et économique, et qui présentaient des profils tout à fait particuliers dans l'analyse des groupes telle que l'analyse conduite en termes de degré d'intensification. Cette approche ouvre des perspectives en termes d'analyse des trajectoires possibles des exploitations à partir de la position moyenne des points pour un thème donné. D'un côté pratique, elle permet aussi d'identifier les variables clés de fonctionnement qui peuvent être la base d'un suivi technico-économique.

Réalisée à partir d'un corpus d'hypothèses de regroupement des exploitations ou de profils fictifs construits par les partenaires, cette approche a permis de discuter, de comprendre et d'analyser les convergences et les divergences des exploitations par rapport aux groupes « fictifs », mais aussi pour chaque thème ou facette de l'exploitation. La validation des groupes par les partenaires donne aujourd'hui une base qui peut être utilisée à terme comme groupes fictifs, c'est-à-dire à partir desquels pourront être déterminées les ressemblances ou les divergences d'un exploitant par rapport à chaque groupe, et ce, pour l'ensemble des exploitations de la Réunion.

\section{Remerciements}

Les auteurs remercient l'ensemble des instituts de développement et partenaires privés qui ont été impliqués dans ce travail, en particulier la coopérative laitière (Sica Lait), l'Union des associations foncières pastorales, l'Etablissement département de l'élevage, la Daf, l'Urcoopa et les centres de gestion. Sont remerciés tout spécialement Mmes Evenat et Bigot de la Sica Lait, pour leur appui permanent à la réalisation de ce travail, ainsi que l'ensemble des éleveurs enquêtés pour leur disponibilité et leur confiance.

\section{BIBLIOGRAPHIE}

1. ALARY V., MESSAD S., TILLARD E., 2001. Approche fonctionnelle de la diversité des systèmes d'élevage laitiers à l'île de la Réunion. Utilisation de I'Afm (analyse factorielle multiple) comme aide à I'interprétation de la variabilité inter et intra-groupe. In : $8^{\text {es }}$ Rencontres autour des recherches sur les ruminants. Paris, France, Institut de l'élevage, p. 251.

2. BLANFORT V., THOMAS P., FONTAINE O., RIVIERE E., 2000. La gestion agroécologique des prairies. In : Mandret. G. coord., L'élevage bovin à la Réunion. Synthèse de quinze ans de recherche. Montpellier, France, Cirad, p. 129-160. (Coll. Repères)

3. BROSSIER J., 1987. Système et système de production. Note sur ces concepts. Cah. Sci. Hum., 23 : 377-390.

4. CAPILLON A., 1993. Typologie des exploitations agricoles, contribution à l'étude régionale des problèmes techniques, 2 tomes. Thèse Doct., INA PG, Paris, France, 48 et 301 p.

5. CAPILLON A., SEBILLOTTE M., THIERRY J., 1975. Evolution des exploitations d'une petite région. Elaboration d'une méthode d'étude. Paris, France, Cnasea Geara, 35 p.

6. CHAMBERS J.M., HASTIE T.J., 1993. Statistical models in S. London, UK, Chapman and Hall, p. 309-376.

7. CHESSEL D., THIOULOUSE J., DOLEDEC S., OLIVIER J.M., 1996 Fiches modules $\mathrm{V}$ : K-tableaux. In : Analyses multivariées et expression graphique des données environnementales. Lyon, France, Université Lyon I, Documentation de la programmathèque ADE-4, 28 p.

8. CRISTOFINI B., 1986. La petite région vue à travers le tissu de ses exploitations : un outil pour l'aménagement et le développement rural. Paris, France, Inra, 48 p. (Etudes et recherches $n^{\circ} 6$ )

9. ESCOFIER B., PAGES J., 1983. Méthodes pour l'analyse de plusieurs groupes de variables. Application à la caractérisation des vins rouges du Val de Loire. Revue Stat. appl., 31 : 43-59.
10. ESCOFIER B., PAGES J., 1984. L'analyse factorielle multiple : une méthode de comparaison de groupes de variables. In : Diday E. et al. Eds, Data analysis and informatics III. Amsterdam, The Netherlands, Elsevier, p. 41-55.

11. ESCOFIER B., PAGES J., 1986. Le traitement des variables qualitatives et des tableaux mixtes par analyse factorielle multiple. In : Diday E. et al. Eds, Data analysis and informatics IV. Amsterdam, The Netherlands, Elsevier, p. 179-191.

12. ESCOFIER B., PAGES J., 1998. Analyses factorielles simples et multiples. Objectifs, méthodes et interprétation, $3^{\mathrm{e}}$ edn. Paris, France, Dunod.

13. FAYE B., GRELET Y., 1990. Type d'élevage et profil de santé. Deux stratégies statistiques. In : Actes colloque Agro-industrie et méthodes statistiques, Association pour la statistique et ses utilisations, Angers, France, 14-15 juin 1990, p. 111-125.

14. FAYE B., LHOSTE P., 1999. Le conseil en élevage en milieu tropical. In : Actes $6^{\text {es }}$ Rencontres autour des recherches sur les ruminants, Paris, France, 1999, p. 63-67.

15. Groupe d'étude et de réflexion interrégional, 1996. L'analyse des données évolutives, méthodes et applications. Paris, France, Technip.

16. JOUVE P., 1984. Le diagnostic agronomique préalable aux opérations de recherche-développement. In : Diagnostics sur le fonctionnement des systèmes agraires. Cah. Rech. Dév., n 3-4 : 67-76.

17. LANDAIS E., 1996. Typologies d'exploitations agricoles. Nouvelles questions, nouvelles méthodes. Econ. Rurale, n $236: 3-15$.

18. LEBART L., MORINEAU A., PIRON M., 1993. Statistique exploratoire multidimensionnelle. Paris, France, Dunod.

19. MANRINQUE, OLAIZOLA A.M., BERNUES A., MAZA M.T., SAEZ A., 1999. Economic diversity of farming systems and possibilities for structural adjustment in mountain livestock farms. In : Options méditerranéennes, Série B : Etudes et recherches, $\mathrm{n}^{\circ} 27$, Systèmes d'élevage et gestion de l'espace en montagnes et collines méditerranéennes. Montpellier, France, Ciheam, p. 81-94.

20. MARCHAL V., DELZESCAUX D., LHOSTE P., 1992. La diversité des systèmes d'élevage bovins allaitants en province du nord de Nouvelle Calédonie. Cah. Rech. Dév., n 32-2 : 31-37.

21. MONICAT F., BORNE P.M., MARON P., 1992. Zonage et typologie des exploitations possédant des petits ruminants dans les systèmes de production traditionnels du Zimbabwe. Cah. Rech. Dév., n 32-2 : 38-56.

22. ORSINI J.P.G., LHOSTE P., BOUCHIER A., FAYE A., NYANG L., 1985. Une typologie d'exploitations agropastorales au Siné-Saloum, Sénégal. Revue Elev. Méd. vét. Pays trop., 38 : 200-210.

23. OSTY P.L., 1978. L'exploitation agricole vue comme un système. Diffusion de l'innovation et contribution au développement. Bull. tech. Inf. min. Agric., $326: 43-49$.

24. PAILLAT J.M., BLANFORT V., 2000. Le fonctionnement des systèmes d'élevage. In : Mandret. G. coord., L'élevage bovin à la Réunion. Synthèse de quinze ans de recherche. Montpellier, France, Cirad, p. 165-176. (Coll. Repères)

25. PERROT C., LANDAIS E., 1993. Comment modéliser la diversité des exloitations agricoles. Cah. Rech. Dév., nº 33 : 24-40

26. PERROT C., LANDAIS E., 1993. Exploitations agricoles : pourquoi poursuivre la recherche sur les méthodes typologiques. Cah. Rech. Dév., $n^{\circ} 33: 13-23$

27. PERROT C., PIERRET P., LANDAIS E., 1995. L'analyse des trajectoires des exploitations agricoles. Une méthode pour actualiser les modèles typologiques et étudier l'évolution de l'agriculture locale. Econ. Rurale, $n^{\circ} 228: 35-47$

28. TACHE C., 2001. Diagnostic des exploitations laitières de l'île de la Réunion. Mémoire de fin d'études, Cergy-Pontoise, France, Istom, 96 p.

29. THIOULOUSE J., CHESSEL D., DOLEDEC S., OLIVIER J.M., 1997. ADE-4: A multivariate analysis and graphical display software. Stat. Comput., 7: 75-83.

30. TILLARD E., LANOT F., BIGOT C.-E., NABENEZA S., PELOT J., 2000. Les performances de reproduction en élevage laitier. In : Mandret. G. coord., L'élevage bovin à la Réunion. Synthèse de quinze ans de recherche. Montpellier, France, Cirad, p. 257-292. (Coll. Repères)

Reçu le 03.09.2001, accepté le 17.09.2003 


\section{Summary}

Alary V., Messad S., Taché C., Tillard E. Approach to the Diversity of Dairy Farm Systems in Reunion

The impacts of economical, institutional and technical changes on farming systems' organization and performance differ between systems depending on initial endowments, projects, organization types and productive-system management, investment opportunities, etc. As it is difficult to apprehend exhaustively individual diversity, farm modeling through typology appears as the only way to approach farming systems' diversity in order to assess change differentiated effects and to ensure that research and development will reflect local needs and diversity. The typology, which characterizes the diversity of dairy farm systems in Reunion, results from an iterative and progressive process that combines principles from the typology à dire $d^{\prime}$ expert (i.e. based on an expert representation of the diversity) and multivariate methods. The typology was developed from surveys and interviews of farmers, and the various actors of the dairy sector: cooperatives, development agencies, local administrators, management centers, etc.

Key words: Classification - Farming system - Factorial analysis - Animal husbandry - Milk - Reunion.

\section{Resumen}

Alary V., Messad S., Taché C., Tillard E. Enfoque sobre la diversidad de los sistemas de cría lechera en la Reunión

Los efectos de los cambios en el medio económico, legal y técnico de las explotaciones sobre el funcionamiento y el rendimiento de éstas difieren en función de las posibilidades iniciales de los productores, de sus proyectos, del tipo de organización y de conducta de los sistemas productivos, de las posibilidades de inversión, etc. Sin embargo, es difícil comprender la complejidad de los casos y la sola construcción de una tipología puede permitir la comprensión de la diversidad de las explotaciones para relacionar los efectos diferenciados de estos cambios, así como ajustar la investigación y el desarrollo a las variadas necesidades y estructuras en un territorio definido. La tipología realizada para caracterizar la diversidad de las explotaciones lecheras en la Reunión resulta de un proceso de construcción interactivo, que se apoya sobre ciertos principios de la topología dicha de experto, recurriendo a métodos multivariados. Esta topología se construye sobre la base de encuestas y de entrevistas llevadas a cabo con los productores y con los diferentes participantes de la filial: cooperativas, agentes de desarrollo, autoridades locales, centro de gestión.

Palabras clave: Clasificación - Sistema de explotación Análisis factorial - Ganadería - Leche - Réunión. 